\title{
New light on the X-ray spectrum of the Crab Nebula
}

\author{
R. Willingale ${ }^{1}$, B. Aschenbach ${ }^{2}$, R. G. Griffiths ${ }^{1}$, S. Sembay $^{1}$, R. S. Warwick ${ }^{1}$, W. Becker ${ }^{2}$, A. F. Abbey ${ }^{1}$, \\ and J.-M. Bonnet-Bidaud ${ }^{3}$ \\ 1 Department of Physics and Astronomy, University of Leicester, University Road, Leicester LE1 7RH, UK \\ 2 Max-Planck-Institut für extraterrestrische Physik, Giessenbachstraße, 85740 Garching, Germany \\ 3 Service d'Astrophysique DAPNIA/SAp, CE Saclay, 91191 Gif-sur-Yvette, France \\ e-mail: rw@star.le.ac.uk; bra@mpe.mpg.de; rgg@star.le.ac.uk; sse@star.le.ac.uk; \\ rsw@star.le.ac.uk; web@me.mpg.de; afa@star.le.ac.uk; bobi@discovery.saclay.cea.fr
}

Received 2 October 2000 / Accepted 6 November 2000

\begin{abstract}
XMM-Newton observations of the Crab provide new information on its integrated X-ray spectrum and the variation of the spectral form across the nebula. The Crab pulsar and its surrounding torus exhibit the hardest spectra with power-law indices of $\Gamma=1.6$ and 1.8. The jet and outer reaches of the nebula are significantly softer with $\Gamma=2.1$ and 2.3 respectively. For the whole nebula, the huge number of recorded counts allows a detailed examination of the soft X-ray absorption due to cool gas in the foreground of the Crab. Absorption edges due to oxygen and neon are clearly identified. Oxygen and iron in the interstellar medium are underabundant by a factor of $0.63 \pm 0.01$. The average $N_{\mathrm{H}}=3.45 \pm 0.0210^{21} \mathrm{~cm}^{-2}$ and varies by less than $\pm 11 \%$ on a scale equal to or larger than 20 arcsec over the face of the nebula. These observations of the Crab provide an excellent demonstration of the power of the EPIC cameras on XMM-Newton for spatial, spectral and timing studies.
\end{abstract}

Key words. ISM: supernova remnants - ISM: individual: Crab Nebula

\section{Introduction}

The Crab Nebula is the remnant of the historically recorded supernova of $\mathrm{AD} 1054$. It is powered by the central $33 \mathrm{~ms}$ Crab pulsar, which is accelerating electrons to highly relativistic energies. The electrons propagating through the nebula and its magnetic field lose energy by emitting synchrotron radiation which is observed throughout the electromagnetic spectrum from low frequency radio up to very hard X-rays. A search for spatial variations of the synchrotron spectrum may help us to understand the details of the injection and transport mechanisms of electrons into and through the nebula. Recently Bietenholz et al. (1997) have summarized the status at radio frequencies coming to the conclusion that there are no significant changes in the radio spectral index over the nebula. Scargle (1969) reported a slight steepening of the optical continuum spectrum towards the North-Western boundary of the nebula. Using CCD measurements Véron-Cetty \& Woltjer (1993) showed that the optical spectral index changes considerably from place to place over the nebula. In general, the spectrum is flattest in the region close to and northwest of the pulsar and steepens by $\sim 0.5$ at the outermost boundary. They concluded that simple interpretations involving constant

Send offprint requests to: R. Willingale injection of relativistic electrons combined with energy loss due to synchrotron radiation are inadequate to explain the data. Spatially resolved X-ray spectra might shed further light on this problem.

The first X-ray image was obtained with the EinsteinHRI (Harnden \& Seward 1984), although earlier lunar occultation measurements (for a summary see Aschenbach \& Brinkmann 1975) had already revealed the extent of the Crab Nebula and the off-set between the pulsar and the position of the maximum X-ray surface brightness. The ROSAT-HRI image (Aschenbach 1992; Hester et al. 1995) gave improved spatial resolution and contrast but like the Einstein measurements lacked spectral information. Weisskopf et al. (2000) have presented a superb X-ray image of the Crab Nebula revealing astonishing details. They also discovered spectral variations across the Crab Nebula derived from hardness ratio changes which, however, are difficult to convert into spectral parameters. Here we report the results of spectral analysis of the entire Crab performed with the EPIC MOS CCDcameras on board XMM-Newton, which for the first time allows us to determine the level and the spectral index of the continuum as well as the absorption column density characteristics. The continuum spectral changes correlate with known features in the continuum image, the torus 


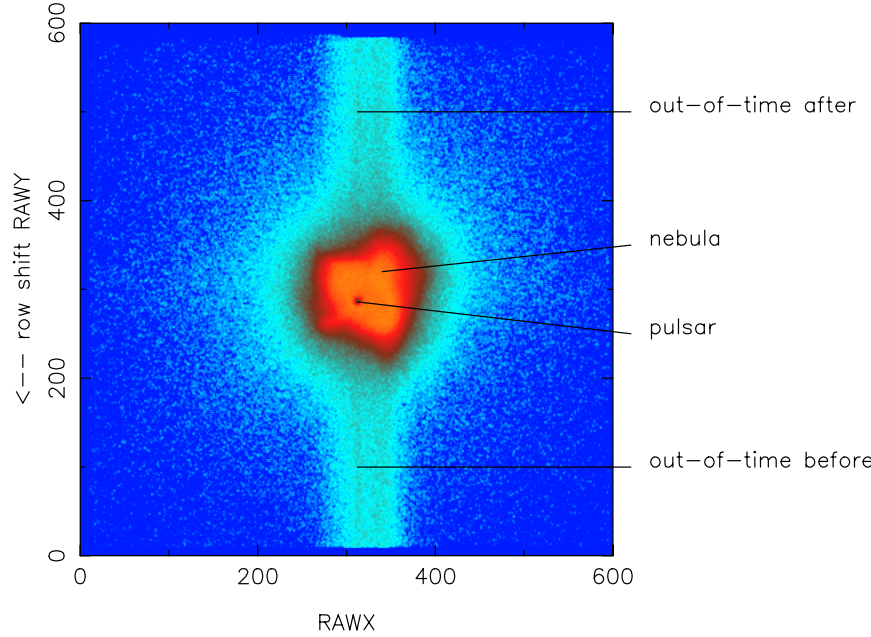

Fig. 1. The raw image recorded on MOS 1

(Aschenbach \& Brinkmann 1975), the jet and counterjet (Brinkmann et al. 1985) as well as the outskirts.

\section{Observations}

The Crab Nebula was observed by XMM-Newton during the PV/CAL phase of the mission, revolution 56, 2000-0329 19:56:51 to 2000-03-30 02:05:14. Details of the XMMNewton mission and the EPIC MOS cameras can be found in Jansen et al. (2001) and Turner et al. (2001). The thin filter was selected for both EPIC MOS cameras. The outer MOS CCDs were in normal imaging mode with a frame time of $2.9 \mathrm{~s}$ whereas the central CCD chips of both MOS 1 and MOS 2 were in refreshed frame store mode in which the frame exposure time is just $200 \mathrm{~ms}$. This fast mode can help to reduce event pile-up for bright sources whilst retaining a full imaging capability. Immediately before and after each $200 \mathrm{~ms}$ integration the full image area of 602 rows is shifted at $15 \mu$ s per row (taking $9 \mathrm{~ms}$ ) into the frame store area of the chip. During these periods socalled out-of-time events from the central source are detected in low rows (before) and in high rows (after). After the image from the $200 \mathrm{~ms}$ integration has been shifted into the frame store it is read out slowly pixel by pixel to extract the event data in the normal way. The full frame cycle is repeated every $2.9 \mathrm{~s}$ so the exposure efficiency is only $6.9 \%$. Furthermore, since the event rate from the Crab caused saturation of the telemetry, only about $50 \%$ of the central chip frames could be collected. In the event an elapsed time of $22000 \mathrm{~s}$ allowed 3324 frames to be recorded from MOS 1 and 3536 frames from MOS 2, giving a total of 1372 seconds exposure time. Figure 1 shows the raw image recorded by MOS 1 . The pixel size is $40 \mu \mathrm{m}$ corresponding to 1.1 arcsec and the sky area covered is $11 \times 11$ square arcmin. The maximum count per CCD pixel per frame recorded near the centre of the nebula is $3.610^{-2}$ resulting in $1-2 \%$ of the event pattern types $0-12$ (good events) being multiple (piled-up). Flux loss due to pile-up is more serious. Of order $30 \%$ of events form complicated multiple patterns which are rejected on-board. The event
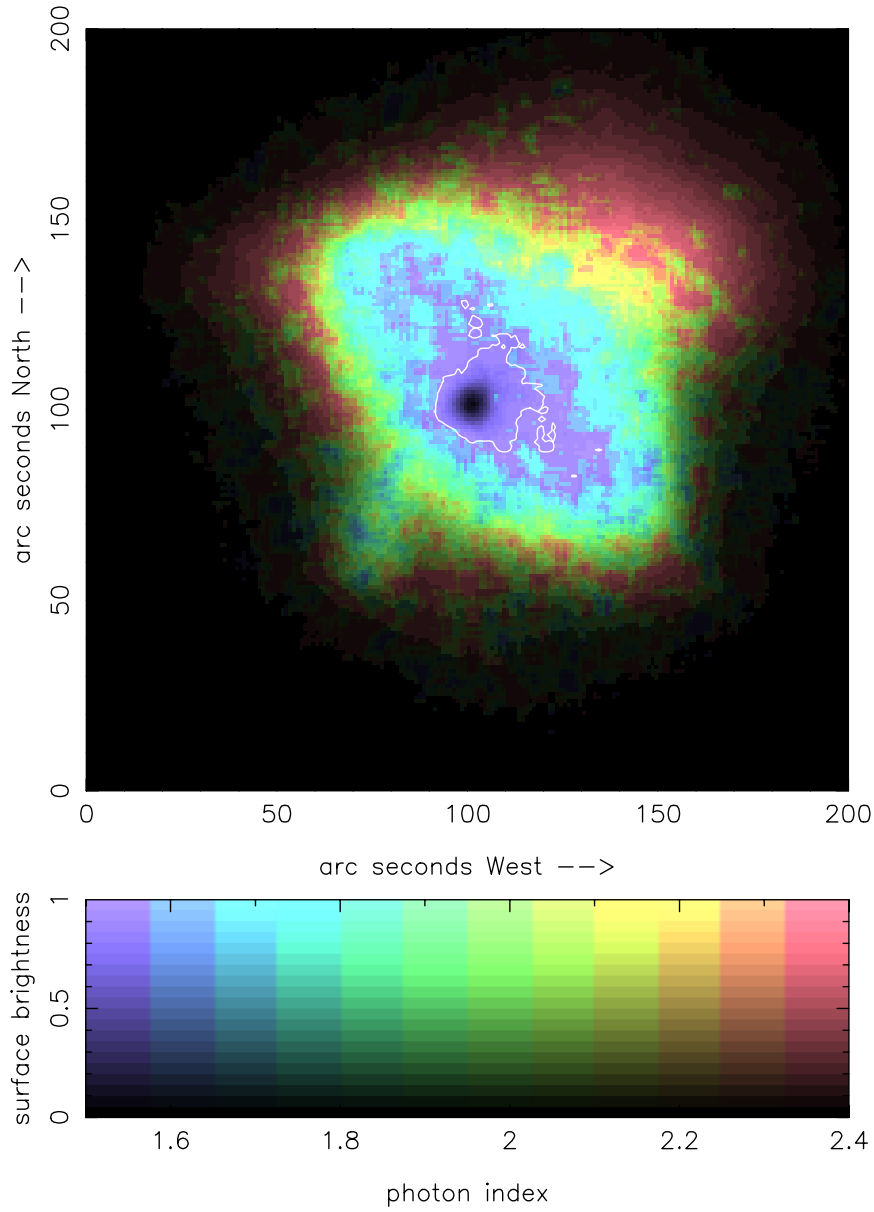

Fig. 2. X-ray colour image in 1 arcsec pixels. The colour coding of surface brightness and photon index is shown in the lower panel. The white contour encircles the region compromised by pile-up

density at the pulsar is much higher and within a radius of $\approx 15$ arcsec most of the events are recognized as multiple and lost.

\section{An X-ray colour image of the Crab Nebula}

Figure 2 shows the X-ray colour image. This was generated using two images, the first calculated by summing the events in the energy band $0.3-10.0 \mathrm{keV}$ from MOS 1 and MOS 2 in 1 arcsec pixels and the second calculated from the ratio of events $(3.1-10.0 \mathrm{keV}) /(2.0-3.1 \mathrm{keV})$ in 5 arcsec pixels. The hardness ratio was converted into a photon index assuming a power-law continuum and using the instrument response function. Figure 2 is displayed using 20 intensity levels and colour coded with purple equivalent to a photon index of 1.5 through to red 2.4. The colour mapping of photon index and surface brightness is shown in the panel below the image. The total number of events is $2.410^{6}$. The region encircled by the white contour is compromised by pile-up. The region at the pulsar is so piled-up that most of the counts from the point source are lost. The spatially resolved features of 

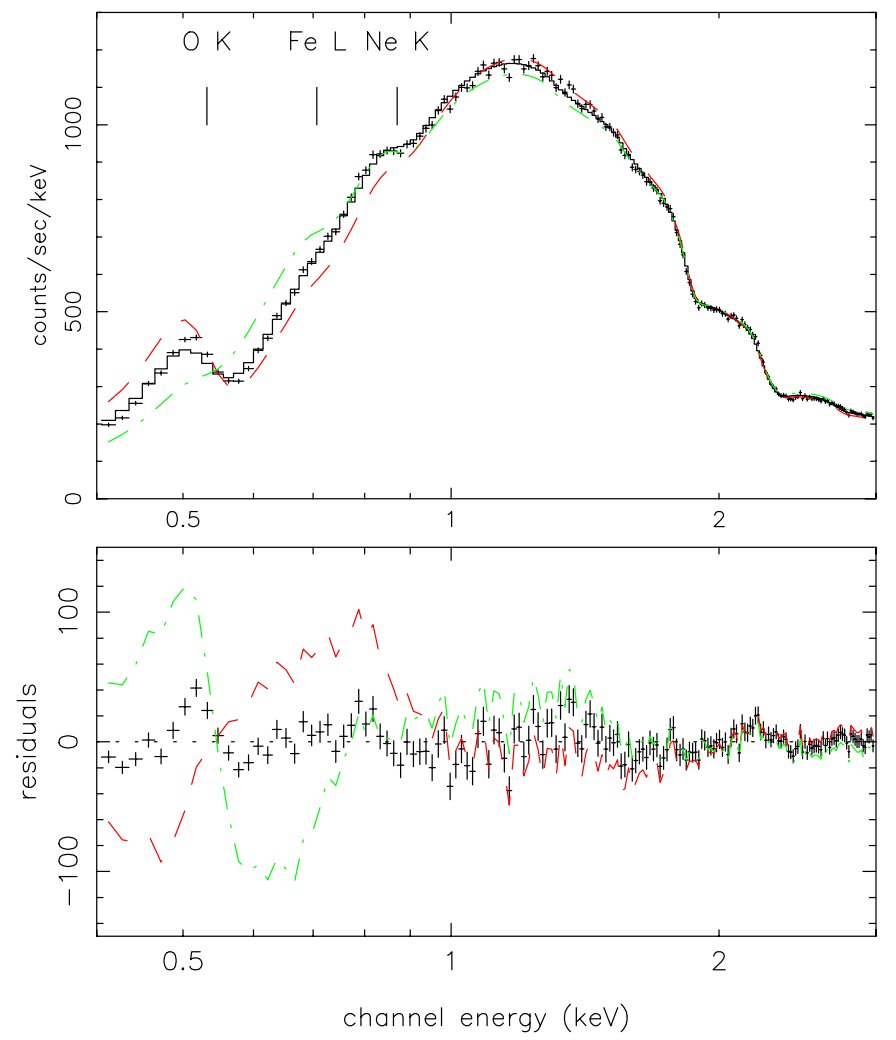

Fig. 3. Interstellar absorption features in the MOS 1 spectrum. Three fits are shown; the best fit with depleted oxygen and iron, the red curve assuming solar abundances and the green curve with the oxygen abundance set to zero

the nebula match well with the high resolution Chandra image (Weisskopf et al. 2000).

\section{Interstellar absorption of the X-ray spectrum}

A spectrum of the whole nebula was accumulated excluding a circular region of radius 15 arcsec surrounding the pulsar. Figure 3 shows the observed spectrum 0.4 to $3.0 \mathrm{keV}$ from MOS 1. The results from MOS 2 are almost identical. The spectrum was fitted with a single powerlaw continuum and interstellar absorption assuming the photoelectric absorption cross-sections from BalucinskaChurch \& McCammon (1992) with the new He crosssection from Yan et al. (1998). The fit above $1.5 \mathrm{keV}$ where the continuum is unabsorbed was excellent. However, below $0.8 \mathrm{keV}$ the residuals with respect to the model count spectrum with $N_{\mathrm{H}} \approx 310^{21} \mathrm{~cm}^{-2}$ became unacceptably large. The extent of the problem can be seen in the residuals plotted in Fig. 3. Potentially the most troublesome feature is the oxygen $\mathrm{K}$ absorption edge in the CCD quantum efficiency at $0.532 \mathrm{keV}$. However, spectral fits on the bright extragalactic sources Mkn 421, 3C 273 and MS 0737.9, for which no significant oxygen $\mathrm{K}$ absorption intrinsic to the source or interstellar column is expected, were used to fine tune the instrumental oxygen $\mathrm{K}$ absorption profile to yield excellent fits in the region of this absorption feature. Also emission line profiles from the on-board calibration source and cosmic sources are well fit by the detector redistribution function. Thus the discrepancy is not instrumental.

If the older photoelectric absorption data from Morrison \& McCammon (1983), in which the oxygen K cross-section is lower, were used the fit was found to be considerably better indicating that the spectral fitting was sensitive to the modelling of the interstellar absorption. The notch in the observed spectrum near $0.9 \mathrm{keV}$ may be interpreted as due to neutral interstellar neon $\mathrm{K}, 0.870 \mathrm{keV}$. If the abundance of neon is set to zero this notch disappears from the predicted count spectrum. Similarly the large notch near $0.5 \mathrm{keV}$ is due to absorption by interstellar oxygen $\mathrm{K}$ at $0.532 \mathrm{keV}$. The green dashdot curve is the best fit model spectrum with the oxygen abundance set to zero; in this case the notch has all but disappeared. As indicated on the plot, Fe L absorption features at $0.707,0.720$ and $0.845 \mathrm{keV}$ also influence the predicted count spectrum although a distinct iron notch is not visible. The step features at higher energies in the count spectrum near 1.9 and $2.2 \mathrm{keV}$ are the Si K absorption edge at $1.847 \mathrm{keV}$ in the detector quantum efficiency and the series of gold $\mathrm{M}$ absorption edges in the mirror reflectivity starting at $\mathrm{M} \mathrm{V}, 2.242 \mathrm{keV}$. The very good counting statistics in this Crab spectrum allowed fine adjustment of these instrumental features. Small changes of order $5 \%$ near these edges improved the quality of the fit but had no effect on the values of the best fit model parameters.

The red dashed curve in Fig. 3 is the best fit assuming solar abundances from Anders \& Grevesse (1989) for which the predicted O K and Fe L absorption features are both too large. The solid histogram is the best fit with depleted oxygen and iron. Fits were performed with both the oxygen and iron abundance as free parameters but the result was the same as when these two parameters were tied together. An acceptable fit might be obtained by changing the photoelectric cross-sections rather than altering the abundances. However the fact that both the oxygen and iron abundance are depleted by the same amount gives some confidence that the cross-sections are not to blame. The residuals near the $\mathrm{O} \mathrm{K}$ edges are still a little large in the best fit and the reduced chi-squared is 2.3 with no systematic errors included.

The best fit value for the oxygen-iron abundance is $0.63 \pm 0.01$ solar for a line of sight effective hydrogen density of $3.45 \pm 0.0210^{21} \mathrm{~cm}^{-2}$. The errors quoted here and below are $90 \%$ confidence limits assuming one free parameter in the chi-squared fitting. If solar abundances are used then the best fit column density is lower, $3.28 \pm 0.0210^{21} \mathrm{~cm}^{-2}$, which is consistent with the canonical value of $310^{21} \mathrm{~cm}^{-2}$ for the Crab from early X-ray observations (see for example Charles et al. 1979) and the most recent value of $3.23 \pm 0.0210^{21} \mathrm{~cm}^{-2}$ derived from BeppoSAX LECS data by Massaro et al. (2000). The LECS instrument is unable to resolve the energy band containing $\mathrm{O} \mathrm{K}$ and Fe L absorption features and is therefore insensitive to the abundance of these elements. The higher energy resolution of the MOS cameras gives 
the residuals plotted in Fig. 3 indicating that a column with solar abundances is inadequate to describe the data. Previous attempts to measure the oxygen abundance of the interstellar medium using the Crab Nebula with the Focal Plane Crystal Spectrometer (FPCS) on the Einstein Observatory, Schattenburg \& Canizares (1986), and values reported from HEAO-1 A-2 (Charles et al. 1979) were consistent with solar. Schattenburg and Canizares (1986) give $N_{\mathrm{H}}=3.45 \pm 0.4210^{21} \mathrm{~cm}^{-2}$ with an oxygen abundance of $1.1 \pm 0.3$ times solar. Given the much poorer statistics of the FPCS observation and the updated cross-sections used for the present analysis this result is probably consistent with our findings. The errors we quote for the abundance and column are derived from counting statistics only. The true error will depend on the accuracy of the cross-sections used but this is not considered here.

A search was made for variations in column density over the face of the nebula using 20 by 20 arcsec bins. 54 such bins covering an area of diameter 160 arc seconds contained sufficient counts to give a statistical error of $<5 \%$ per bin. The minimum value was $3.0710^{21} \mathrm{~cm}^{-2}$, the maximum $3.7010^{21} \mathrm{~cm}^{-2}$ and the standard deviation $0.1310^{21} \mathrm{~cm}^{-2}$. No bin was significantly different from the mean of $3.4510^{21} \mathrm{~cm}^{-2}$.

The count spectrum shown in Fig. 3 is slightly distorted by pile-up but the effect is too small to see on the scale of the plot. This introduces a systematic change of $-7.5 \%$ in the value of the power-law index, $-9 \%$ in column density and $-7 \%$ in oxygen-iron abundance. The values quoted above for column density and oxygen-iron abundance are not taken from the fit in Fig. 3 but are the best fit results from spectra without pile-up discussed below.

\section{The pulsar}

The pulsar is too bright and its spin period is too rapid to be observed with a frame time of $200 \mathrm{~ms}$. However during the two short periods before and after the frame exposure, "out-of-time" events are detected from the pulsar and nebula with an effective frame time of just a few ms. These events are not piled-up and provide 1-dimensional imaging. Using the RAWX positions (CCD rather than sky coordinates) the nebula and pulsar events can be partially separated and using the RAWY positions the event arrival times can be accurately reconstructed. During detection of out-of-time events the shift rate is $15 \mu$ s per row. The image of the pulsar is less than 20 rows across (limited by the point spread function of the mirrors) so the arrival times have an accuracy of $\pm 0.15 \mathrm{~ms}$. Only the out-of-time events at the top and bottom of RAWY well away from the central image of the nebula are useful. These regions define two short observation windows, each of $2.8 \mathrm{~ms}$, for every $2.9 \mathrm{~s}$ frame cycle. A total of just $38.11 \mathrm{~s}$ of exposure is accrued from combining MOS 1 and MOS 2 out-of-time data.

A period search was performed on the pulsar outof-time events, folding the arrival times using trial periods, exposure corrected to allow for the phase coverage

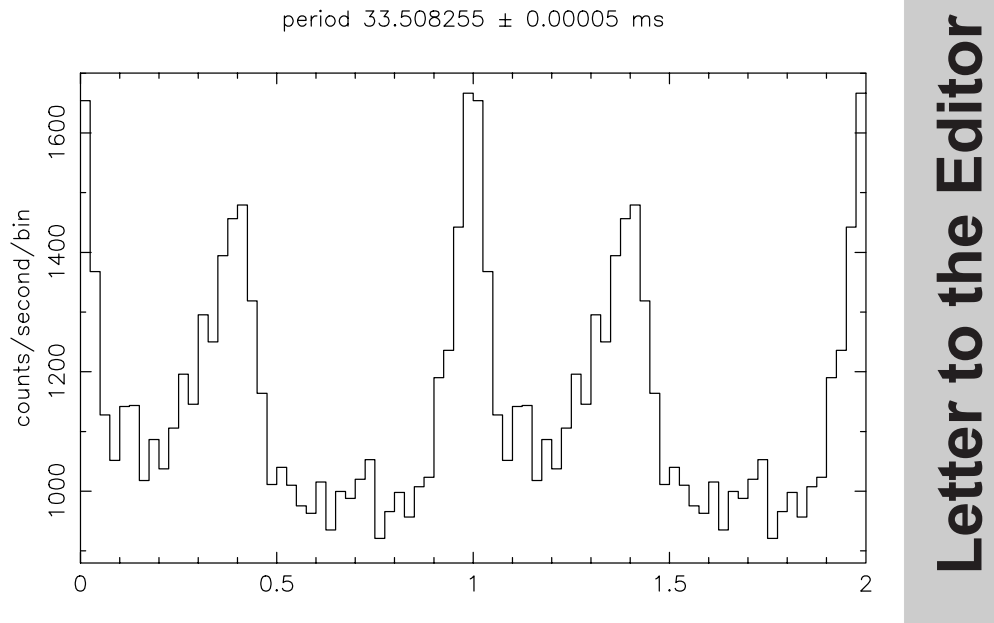

Fig. 4. Pulsar pulse profile for the best fit period MOS $1+2$, corrected for exposure

provided by the many very short exposure windows and using a chi-squared test to look for variation across the trial period. Figure 4 shows the pulse profile for the best fit period. The arrival times were corrected to the Barycentre of the Solar System. Each phase bin has been exposure corrected but in fact the exposure coverage from the many small integration windows is remarkably even. Each bin represents about $1 \mathrm{~s}$ of exposure so typical statistical errors are \pm 30 counts $\mathrm{s}^{-1} \mathrm{bin}^{-1}$. The best fit period is $33.50826 \pm 0.00005 \mathrm{~ms}$ at MJD 51633.0, midnight, 4 hours into the observation. The error corresponds to the full width at base of the chi-squared peak which is consistent with the length of the observation, $22000 \mathrm{~s}$. The published radio monthly ephemeris from Jodrell Bank (Lyne et al.) gives a period of $33.508388 \mathrm{~ms}$ for this epoch so the fractional error in the derived X-ray period is $4.010^{-6}$. The pulse profile is consistent with the statistically much better results obtained from much longer exposures with BeppoSAX, Massaro et al. (2000) and RXTE Pravdo et al. (1997). The XMM-Newton observation tells us nothing new about the pulsar but is a good check on the timing of MOS cameras and the spectrum of the pulsar as discussed below.

\section{The source spectra}

\subsection{Spectral calibration and variation}

The out-of-time events and the outer regions of the nebula image provide clean spectra free from pile-up. Spectra were accumulated from the out-of-time pulsar events, outof-time nebula events, the outer regions of the nebula image (yellow and red in Fig. 2), the harder jet like feature to the South East and the central torus excluding the piledup region near the pulsar. The pulsar events were split between on (phase 0.3-0.47 and 0.9-1.06) and off (phase $0.06-0.3$ and $0.47-0.9$, see Fig. 4 ). The on-range includes the peaks of both pulses which gave the best signal-tonoise in the pulse-phase spectroscopy reported by Massaro et al. (2000). The pulsar (pulsed) spectrum was obtained 
Table 1. The variation in power-law photon index over the Crab Nebula

\begin{tabular}{|l|l|l|}
\hline region & photon index & events \\
\hline pulsar & $1.63 \pm 0.09$ & out-of-time \\
torus & $1.80 \pm 0.006$ & imaged \\
jet & $2.10 \pm 0.013$ & imaged \\
outer nebula & $2.34 \pm 0.005$ & imaged \\
nebula average & $2.108 \pm 0.006$ & out-of-time \\
\hline
\end{tabular}

by subtracting a suitably scaled off-spectrum from the onspectrum. A joint spectral fit was performed with a single column density model including depleted oxygen and iron and a different power-law continuum for each region. The results for the column density model have already been given above. Table 1 summarizes the variation in power-law photon index illustrated in Fig. 2. The reduced chi-squared value for the overall fit was 1.04 with 3853 degrees of freedom and no systematic error included. All the errors quoted for the spectral model parameters are purely statistical and because of the vast number of events involved these errors are small. We can compare the spectral index of the pulsar and the spatial integrated spectrum of the nebula with previous measurements. Both agree very well. The spectral index of the pulsar is $1.63 \pm 0.09$ vs. $1.5 \pm 0.1$ from Toor \& Seward (1977) and $1.60 \pm 0.02$ from the pulse-phase spectroscopy results of Massaro et al. (2000) averaged over the phase range we used. The spectral index for the entire Crab is $2.108 \pm 0.006$ vs. 2.1 from Toor \& Seward (1974) and $2.117 \pm 0.004$ Massaro et al. (2000).

The best estimate of the normalisation of the powerlaw continuum also comes from the fit to the sum of the out-of-time spectra although it is likely that a few percent of flux is lost from the outer region of the nebula in these spectra and that the spectra are contaminated by a few percent with scattered photons from the central nebula image. Not withstanding this the result is $9.59 \pm 0.05$ photons $\mathrm{cm}^{-2} \mathrm{~s}^{-1} \mathrm{keV}^{-1}$ at $1 \mathrm{keV}$. This compares with a value of 9.7 photons $\mathrm{cm}^{-2} \mathrm{~s}^{-1} \mathrm{keV}^{-1}$ given by Woltjer (1987) taken from the original reference of Toor \& Seward (1974). As with other spectral parameters the error quoted for the normalisation is derived from counting statistics. It is likely that the absolute error is of the order \pm 0.5 photons $\mathrm{cm}^{-2} \mathrm{~s}^{-1} \mathrm{keV}^{-1}$.

The ratio of the pulsar flux over that of the Crab Nebula in the $0.4-3.0 \mathrm{keV}$ (the band over which the bulk of the flux is detected by EPIC MOS) is $0.051 \pm 0.001$ estimated from the best period fold Fig. 4 and the fit to the sum of the out-of-time spectra.

\subsection{Discussion}

The canonical photon spectral index of the entire Crab Nebula measured by many experiments is 2.1 , with which our value of $2.108 \pm 0.006$ is fully consistent. In addition to the mean index we find spectral variations across the remnant similar to those derived from the Chandra HETG-ACIS hardness ratio distribution measurements (Weisskopf et al. 2000). We can actually quantify the spectral index. The brightest region of the X-ray image is the so-called torus, which, according to the model of Aschenbach \& Brinkmann (1975), marks the end of the pulsar acceleration zone and the transition into the nebula. Since the acceleration region is not spherically symmetric but confined to a region about $\pm 10^{\circ}$ wide above and below the rotational equatorial plane of the pulsar the emission region appears as a toroid. Since the rotational symmetry axis does not lie in the celestial plane but is tilted by $\theta \sim 30^{\circ}$ the emission region has the observed elliptical shape, with the North-Western part pointing towards the observer. If the electron injection spectrum does not depend on the azimuth of the torus this region should clearly have the flattest spectrum. This is confirmed by our observation (cf. Table 1). It has been suggested by Pelling et al. (1987) that the high brightness of the North-Western part of the torus relative to the low brightness of its opposing part in the South East is due to relativistic beaming or Doppler boosting of the electrons. The boost ratio, which can be estimated from the ratio of the surface brightness $\mathrm{BR}$, depends on the inclination angle $\theta$ of the emission region towards the observer, the photon spectral index $\Gamma$, which we have measured to be 1.8 , and the electron equatorial speed $v_{\mathrm{e}}$. From earlier ROSAT-HRI measurements $\mathrm{BR} \sim 5.5$, so that, given $\theta$ and $\Gamma, v_{\mathrm{e}} \sim 0.34 \times c$. In the framework of the model of Aschenbach \& Brinkmann the jet in the South East and the counterjet towards the North West, which are both clearly visible in the ROSAT (Aschenbach 1992) and Chandra (Weisskopf et al. 2000) images, represent the electron outflow along the spin axis of the pulsar. The jet definitely has an X-ray spectrum steeper than the torus by 0.3 (cf. Table 1 ), which implies that the corresponding electron number spectra at injection differ by 0.6. If relativistic beaming is at work for the torus it should also be applicable for the jet. From the ROSAT observations an upper limit for the surface brightness ratio of 1.1 is derived; for an inclination angle $\theta_{\text {jet }}=$ $60^{\circ}$ and $\Gamma_{\text {jet }}=2.1$ (cf. Table 1 ), $v_{\text {e,jet }}<10000 \mathrm{~km} \mathrm{~s}^{-1}$ or 10 times slower than the speed of the electrons injected into the torus. This speed is still high enough to carry the head of the jet out to the boundary over the lifetime of the Crab, if not decelerated. So the jet speed does not account for the shorter South-Eastern jet. However, if for any reason $v_{\mathrm{e}, \mathrm{jet}} \sim v_{\mathrm{e}}$, then relativistic beaming is not the mechanism which brightens the North-Western part of the torus. Since the region of the jet is relatively close to the pulsar the spectral index difference to the torus is likely to be due to an intrinsically steeper electron spectrum of the jet. The outer regions of the nebula show the steepest spectrum with an index of 2.34. This change seems to follow that observed in the optical (Scargle 1969; Véron-Cetty \& Woltjer 1993), and it is likely to be caused by the lifetime of the corresponding electrons being substantially shorter than the transport time of the electrons to reach the outskirts. For a magnetic field of $630 \mu \mathrm{G}$ 
(Davidson \& Fesen 1985), the synchrotron photon energy of $1 \mathrm{keV}$ corresponds to an electron energy of $7.4 \mathrm{TeV}$. Under these conditions the half life of such electrons is $\sim 4.3$ years, which means that the electron transport speed cannot exceed $0.9 \times c$ because of the spectral steepening observed towards the edges of the nebula. This upper limit is consistent with the maximum electron injection speed into the torus region assuming that Doppler boosting is responsible for the torus brightening. The upper limit of the transport speed of the electrons producing the optical light is a factor of 5 lower using the spectral steepening observed in the optical. Because of the large extent of the X-ray nebula which is comparable to the optical and radio extent there must be some electrons of $\mathrm{TeV}$ energies present at the nebula boundary which puts a lower limit to the transport speed of $1200 \mathrm{~km} \mathrm{~s}^{-1}$. The values given for the upper and lower limit of the electron speed are admittedly crude. A speed as low as $1200 \mathrm{~km} \mathrm{~s}^{-1}$ is clearly much too low because of the very short lifetimes of the electrons but this would only be a problem if the overwhelming majority of the electrons originate from the pulsar rather than being re-accelerated in the Nebula. The observation that the torus and the SouthEastern jet have different X-ray spectra and each have an extent of at least one arcmin indicates that a onedimensional model is inadequate and a two-dimensional approach is required to to explain the X-ray surface brightness seen by Chandra and XMM-Newton.

Comparing Fig. 1 with Fig. 2 in the paper by VéronCetty \& Woltjer (1993) we find a very good correspondence between the optical and X-ray spectral indices. In general the X-ray spectral index appears to be steeper by 0.2 independent of the distance from the pulsar, which is difficult to understand if only electron lifetime in a constant magnetic field are relevant. An analysis of the X-ray data on a finer scale and a comparison with the optical maps will be the subject of a future paper.

\section{Conclusions}

The Crab Nebula is the X-ray astronomer's favourite calibration source. It is bright, stable, has a built in clock and the spectrum is a featureless continuum. The EPIC MOS data accumulated from the full nebula provided a count spectrum with very high precision. The spectrum spanning 1.5 to $5.5 \mathrm{keV}$ was used for fine adjustment of the detector quantum efficiency and the mirror reflectivity.

The high spectral resolution, negligible background, good spatial resolution and large collecting area reveals that the absorbing column is not adequately represented by the standard model using solar abundances. Oxygen and iron appear to be depleted along the line of sight by a factor of 0.63. An absorption feature due to Neon $\mathrm{K}$ is seen and is consistent with solar abundance of this element. The column density is constant to within $\pm 11 \%$ over the nebula on a scale equal to or larger than 20 arcsec.
Using the combination of out-of-time and imaged events we can get a good estimate of the spectra. The spectral index of the pulsar is 1.63 consistent with earlier measurements as is the nebula and pulsar integrated average index of 2.11. The power-law continuum steepens from the central pulsar to the outer edges of the nebula. The bulk of the emission from the inner nebula has a photon index of 1.8 to 2.1 . The outer reaches of the nebula are considerably softer with a photon index of 2.34 .

Acknowledgements. We would like to thank the referee Rino Bandiera for many useful comments and suggestions for improving the paper. The results presented here are based on observations obtained with XMM-Newton, an ESA science mission with instruments and contributions directly funded by ESA Member States and the USA (NASA). EPIC was developed by the EPIC Consortium led by the Principal Investigator, Dr. M. J. L. Turner. The consortium comprises the following Institutes: University of Leicester, University of Birmingham, (UK); CEA/Saclay, IAS Orsay, CESR Toulouse, (France); IAAP Tuebingen, MPE Garching, (Germany); IFC Milan, ITESRE Bologna, OAPA Palermo, Italy. EPIC is funded by: PPARC, CEA, CNES, DLR and ASI.

\section{References}

Anders, E., \& Grevesse, N. 1989, Geochim. Cosmochim. Acta, 53, 197

Aschenbach, B. 1992, Zeiss Info. Jenaer Rundschau, 1, 6

Aschenbach, B., \& Brinkmann, W. 1975, A\&A, 41, 147

Balucinska-Church, M., \& McCammon, D. 1992, ApJ, 400, 699

Bietenholz, M. F., Kassim, N., Frail, D. A., et al. 1997, ApJ, 490, 291

Brinkmann, W., Aschenbach, B., \& Langmeier, A. 1985, Nat, 313,662

Charles, P. A., Kahn, S. M., Bowyer, S., et al. 1979, ApJ, 230, L83

Davidson, K., \& Fesen, R. A. 1985, ARA\&A, 23, 119

Harnden, R. R. Jr., \& Seward, F. D. 1984, ApJ, 283, 279

Hester, J. J., Scowen, P. A., Sankrit, R., et al. 1995, ApJ, 448, 240

Jansen, F., Lumb, D., Altieri, B., et al. 2001, A\&A, 365, L1

Lyne, A. G., Pritchard, R. S., \& Roberts, M. E., WWW, http://www.jb.man.ac.uk/ pulsar/crab.html

Massaro, E., Cusumano, G., Litterio, M., \& Mineo, T. 2000, A\&A, 361, 695

Morrison, R., \& McCammon, D. 1983, ApJ, 270, 119

Pelling, R. M., Paciesas, W. S., \& Peterson, L. E. 1987, ApJ, 319, 416

Pravdo, S. H., Lorella, A., \& Harding, A. K. 1997, ApJ, 491, 808

Scargle, J. D. 1969, ApJ, 156, 401

Schattenburg, M. L., \& Canizares, C. R. 1986, ApJ, 301, 759

Toor, A., \& Seward, F. D. 1974, AJ, 79, 995

Toor, A., \& Seward, F. D. 1977, ApJ, 216, 560

Turner, M. J. L., Abbey, A. F., Arnaud, M., et al. 2001, A\&A, 365, L27

Véron-Cetty, M. P., \& Woltjer, L. 1993, A\&A, 270, 370

Weisskopf, M. C., Hester, J. J., Tennant, A. F., et al. 2000, ApJ, 536, L81

Woltjer, L. 1987, NATO Adv. Sci. Inst., 195, 209

Yan, M., Sadeghpour, H. R., \& Dalgarno, A. 1998, ApJ, 496, 1044 